\title{
Dark matter from a complex scalar singlet and preservation of its stabilising symmetry
}

\section{Leonardo Coito* and Carlos Faubel, Juan Herrero-García and Arcadi Santamaria}

Departament de Física Teòrica, Universitat de València and

IFIC, Universitat de València-CSIC

Dr. Moliner 50, E-46100 Burjassot (València), Spain

E-mail: leocoito@ific.uv.es

This work studies the case where dark matter emerges from a complex scalar field charged under a $U(1)$ global symmetry, which is spontaneously broken. The analysis considers different explicit symmetry breaking terms, motivated by discrete symmetries, and the results show that in some regions of the parameter space these scenarios may be distinguished by combining different observables, such as direct detection and collider signatures. It is also discussed the case where the stabilising symmetry could be spontaneously broken.

Particles and Nuclei International Conference

Lisbon, Portugal

5-10 September 2021

${ }^{*}$ Speaker 


\section{Introduction}

We consider a model where dark matter (DM) [1] emerges from a complex scalar field, $S=\rho+i \theta$, charged under a global $U(1)$ symmetry. This is among the simplest model beyond having the SM and a real scalar singlet, which is very restrained to explain DM [2-5].

From the $U(1)$ the following symmetry breaking (SB) pattern can develop:

- Spontaneously SB (SSB) at a scale $v_{s}$ opens the possibility for having a massless Goldstone boson $(\theta)$, which has a suppressed scattering with SM particles at low energies, however there is no DM candidate.

- Explicit SB gives mass to $\theta$, allowing it to be the DM. In the soft SB limit the DM would be a pseudo-Nambu-Goldstone boson.

The addition of SSB to the model helps preventing it from being over-restrained and suffer instability problems. Explicit SB can be developed from the following subset of discrete symmetries of the kinetic term in the Lagrangian ${ }^{1}$ :

$$
Z_{2}: S \rightarrow-S, \quad Z_{3}: S \rightarrow e^{2 \pi i / 3} S, \quad Z_{4}: S \rightarrow i S, \quad D C P: S \rightarrow S^{*}
$$

An easy long-lived particle can be set by enforcing a discrete symmetry and DCP is the only remaining symmetry after having SSB. Imposing it needs for all the couplings to be real and it also can be identified with a "dark" CP symmetry. The rest of the discrete symmetries, $Z_{i}$, play the role of simplifying the Lagrangian and/or motivate the DCP.

\section{Objectives}

Initially we are going to consider the following models, which we called minimal models (MM), containing one single SB term which satisfies different discrete symmetries $Z_{i}$, and then determine whether they lead to different subsets of the parameter space guarantying a DM candidate:

$$
\begin{array}{rlrl}
V_{1} & =\frac{1}{2} \mu^{3} S+\text { H.c. } & V_{Z_{3}} & =\frac{1}{2} \mu_{3} S^{3}+\text { H.c. }, \\
V_{Z_{2}} & =\frac{1}{2} \mu_{S}^{2} S^{2}+\text { H.c. } \quad V_{Z_{4}}=\frac{1}{2} \lambda_{4} S^{4}+\text { H.c. } .
\end{array}
$$

The MM named $V_{1}$ is the exception here given that it just satisfies DCP - as all the others do. The $V_{1}, V_{Z_{2}}, V_{Z_{3}}$ and $V_{Z_{4}}$ models are going to be denominated as linear, quadratic, cubic and quartic models respectively. The following study assumes the complex field is in thermal equilibrium with the SM in the Early Universe, until later on when it freezes-out because the interactions become insufficient.

\section{Results}

There are four different scenarios where the right value for the relic abundance is reached, which depends on the relative masses of the scalars $\rho, h$ and the DM:

- Resonance with the other scalars (res), occurring for $2 m_{\theta} \simeq m_{\rho, h}$.

${ }^{1}$ Considering only renormalizable operators. 


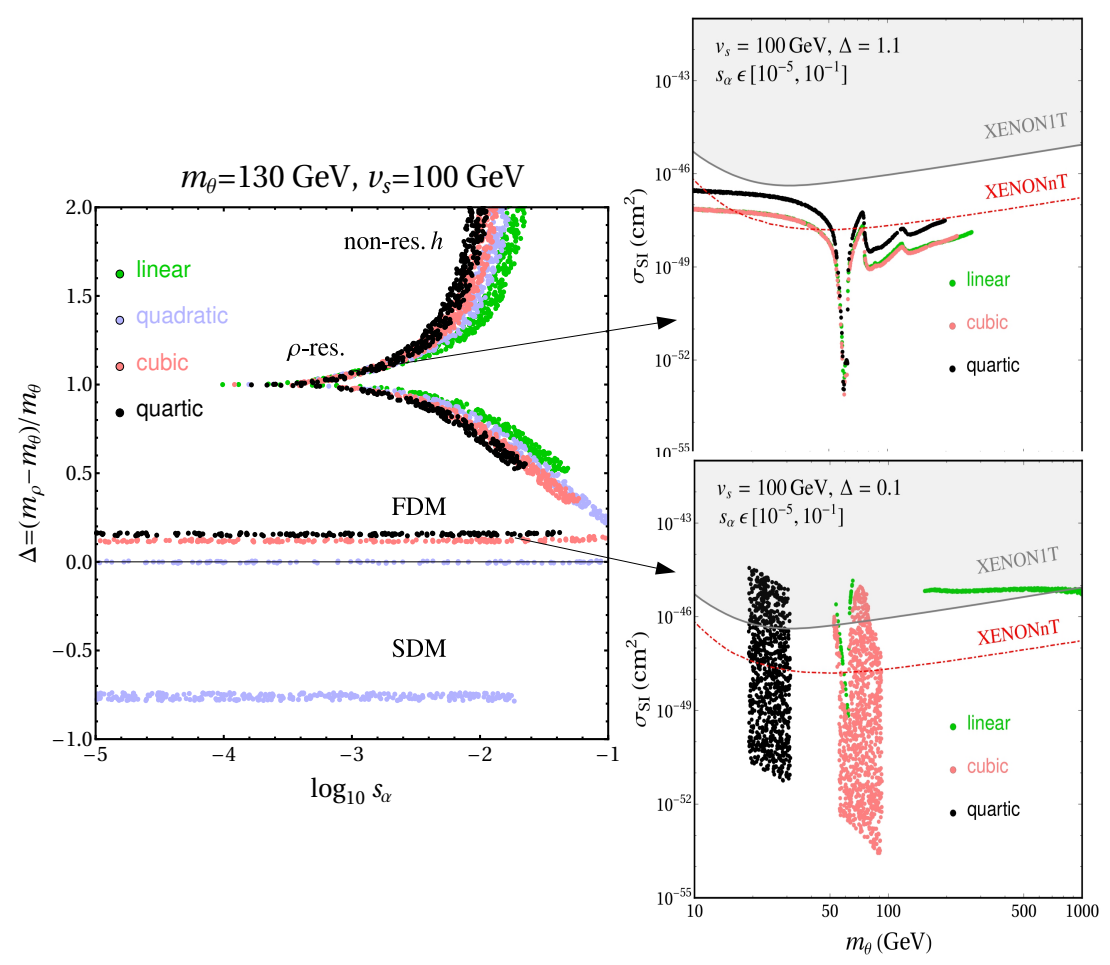

Figure 1: The left panel corresponds to the scan on (logarithm of) the sine of the mixing angle $s_{\alpha}$ and the normalized mass splitting $\Delta$ with fixed $v_{s}=100 \mathrm{GeV}$ and for the MMs described. XENON1T [6] and invisible Higgs decay constraints were imposed. The right panels show the resulting DD cross section as a function of $m_{\theta}$ for mixing angles belonging to the interval $\left[10^{-5}, 10^{-1}\right]$ and two illustrative values of $\Delta=0.1,1.1$.

- Secluded DM (SDM) for lighter pairs of scalars: $m_{\theta} \gtrsim m_{\rho, h}$.

- Forbidden DM (FDM) for slightly heavier scalars $m_{\rho, h} \gtrsim m_{\theta}$.

- Non-resonance (non-res) Higgs-mediated annihilations to SM particles for $m_{\theta} \gtrsim 100 \mathrm{GeV}$ and somewhat large mixing angles.

The regions of parameter space that meet the requirements of reproducing the right relic abundance $^{2}$ and avoiding direct (DD) and indirect (ID) detection constraints are shown in Fig. 1 (top panel) for $m_{\theta}=130 \mathrm{GeV}$. The scan was made in the normalized mass splitting parameter $\Delta$ and the mixing angle for a fixed value of the VEV $\left(v_{s}=100 \mathrm{GeV}\right)$. In conclusion the MMs can be disentangle just when the relic abundance is reached under the FDM and SDM scenarios. In the bottom of Fig. 1 it is shown the resulting DD cross section for different values of masses and the superimposed mixing angles. This indicates that a positive signal from DD or ID measurements might reveal some possible symmetry pattern.

\section{Beyond the minimal models}

Secondly, we desire to go beyond the MMs to consider their combinations in pairs of SB terms. However, two real SB terms may lead to SSB of the DCP symmetry [7] and the instability of the

\footnotetext{
${ }^{2}$ The values were set to $0.5 \leqslant \Omega / \Omega_{\text {obs }} \leqslant 1$, with $\Omega_{\text {obs }} h^{2}=0.120 \pm 0.001$, to make the regions clearly visible.
} 
DM. Consider in general the SB potential:

$$
V_{S B}=a S^{n}+b S^{n^{\prime}}+\text { H.c. },
$$

where $a$ and $b$ can be expressed as $\mu^{3} /\left(\sqrt{2} v_{s}^{3}\right), \mu_{s}^{2} /\left(2 v_{s}^{2}\right), \mu_{3} /\left(2 \sqrt{2} v_{s}\right)$ and $\lambda_{4} / 4$ for the linear, quadratic, cubic and quartic models respectively. In being cautious about not breaking the DCP symmetry, we consider as a theoretical constraint to remain in the colored regions shown in Fig. 2. This was done by asking the VEV to be real and, without loss of generality, positive: $\langle S\rangle=\left\langle S^{*}\right\rangle>0$.

The new scans show that when combining pairs of MMs the allowed regions of parameter space are only expanded in between the originals. As an example, the results were plotted in the case of taking the cubic and quartic potentials together, see Fig. 2.
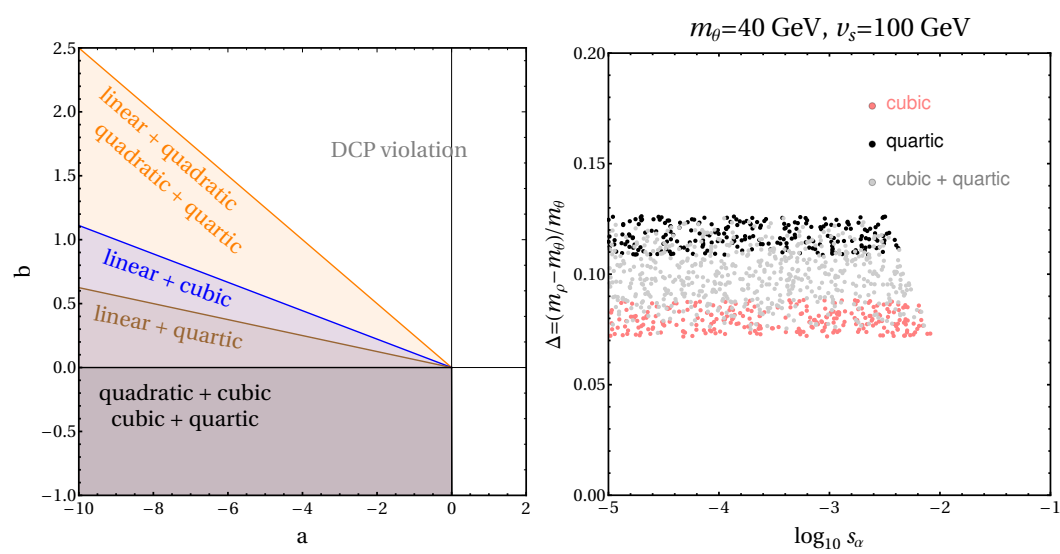

Figure 2: Left) Upper limits on the SB terms $a$ and $b$ for the conservation of DCP in all the possible combinations of two MMs. Without loss of generality $n<n^{\prime}$. Right) Scan for the cubic and quartic models in $\Delta$ and $s_{\alpha}$ around the region of $\Delta \gtrsim 0$ (FDM). The points in gray correspond to those which satisfy requirements for DM and the preservation DCP.

\section{Acknowledgement}

Work partially supported by the FEDER/MCIyU-AEI grant FPA2017-84543-P, the "Generalitat Valenciana" under grant PROMETEO/2019/087 and by the AEI-MICINN under grant PID2020113334GB-I00/AEI/10.13039/501100011033.

\section{References}

[1] L. Coito, C. Faubel, J. Herrero-Garcia and A. Santamaria 2106.05289.

[2] C. P. Burgess, M. Pospelov and T. ter VeldhuisNucl. Phys. B 619 (2001) 709-728, [hep-ph/0011335].

[3] A. Beniwal, J. Herrero-García, N. Leerdam, M. White and A. G. WilliamsJHEP 21 (2020) 136, [2010.05937].

[4] GAMBIT collaboration, P. Athron et al.Eur. Phys. J. C 79 (2019) 38, [1808 . 10465].

[5] J. M. Cline, K. Kainulainen, P. Scott and C. WenigerPhys. Rev. D 88 (2013) 055025, [1306.4710].

[6] XENON collaboration, E. Aprile et al.Phys. Rev. Lett. 121 (2018) 111302, [1805. 12562].

[7] H. E. Haber and Z. SurujonPhys. Rev. D 86 (2012) 075007, [1201. 1730]. 